\title{
ON NONATOMIC BANACH LATTICES AND HARDY SPACES
}

\author{
N. J. KALTON AND P. WOJTASZCZYK \\ (Communicated by Dale Alspach)
}

\begin{abstract}
We are interested in the question when a Banach space $X$ with an unconditional basis is isomorphic (as a Banach space) to an order-continuous nonatomic Banach lattice. We show that this is the case if and only if $X$ is isomorphic as a Banach space with $X\left(\ell_{2}\right)$. This and results of Bourgain are used to show that spaces $H_{1}\left(\mathbf{T}^{n}\right)$ are not isomorphic to nonatomic Banach lattices. We also show that tent spaces introduced by Coifman, Meyer, and Stein are isomorphic to $\operatorname{Rad} H_{1}$.
\end{abstract}

\section{INTRODUCTION}

There is a natural distinction between sequence spaces and function spaces in functional analysis; as an example, let us point out the subtitles of two volumes of [15] and [16]. In this paper we use the term sequence space to indicate a space with the structure of an atomic Banach lattice and the term function space to indicate a space with the structure of a nonatomic Banach lattice. Many classical function spaces (e.g., the spaces $L_{p}[0,1]$ for $1<p<\infty$ [22] or [16]) have unconditional bases and hence are isomorphic as Banach spaces to sequence spaces (atomic Banach lattices). On the other hand, $L_{1}[0,1]$ has no unconditional basis ([22] or [16]) and in the other direction the sequence spaces $\ell_{p}$ for $p \neq 2$ are not isomorphic to any nonatomic Banach lattice [1]. In this note we discuss a general criterion for deciding whether a Banach space with an unconditional basis (i.e., a sequence space) can be isomorphic to a nonatomic Banach lattice (i.e., a function space). Our main result (Theorem 2.4) gives a simple necessary and sufficient condition for an atomic Banach lattice $X$ to be isomorphic to an order-continuous nonatomic Banach lattice; of course, if $X$ contains no copy of $c_{0}$, every Banach lattice structure on $X$ is order-continuous.

Our main motivation is to study the Hardy space $H_{1}(\mathbf{T})$. After the discovery that the space $H_{1}(\mathbf{T})$ has an unconditional basis [17] it becomes natural to

Received by the editors June 5, 1992.

1991 Mathematics Subject Classification. Primary 46B42, 42B30.

Key words and phrases. Order-continuous Banach lattice, Hardy spaces.

The first author was supported by NSF-grant DMS-8901636; the second author was supported by $\mathrm{KBN}$-grant 2-1055-91-01. This work was done while the second author was visiting the University of Missouri-Columbia; he would like to express his gratitude to the whole Abstract Analysis Group of this department for their hospitality. 
investigate if $H_{1}(\mathbf{T})$ is isomorphic to a nonatomic Banach lattice. Applying Theorem 2.4 to $H_{1}$ and using some previous results of Bourgain [2,3] we show that $H_{1}$ is not isomorphic to any nonatomic Banach lattice and furthermore that $H_{1}\left(\mathrm{~T}^{n}\right)$ is not isomorphic to a nonatomic Banach lattice for any natural number $n$.

We conclude by showing that the space $\operatorname{Rad} H_{1}$ or $H_{1}\left(\ell_{2}\right)$ is isomorphic to the tent spaces $T^{1}$ introduced by Coifman, Meyer, and Stein [4].

\section{LATTICES WITH UNCONDITIONAL BASES}

Our terminology about Banach lattices will agree with [16]; we also refer the reader to $[9,10]$ for the isomorphic theory of nonatomic Banach lattices.

A (real) Banach lattice $X$ is called order-continuous if every order-bounded increasing sequence of positive elements is norm convergent. Any Banach lattice not containing $c_{0}$ is automatically order-continuous.

For any order-continuous Banach lattice $X$ we can define an associated Banach lattice $X\left(\ell_{2}\right)$ (using the Krivine calculus [16, pp. 40-42]) as the space of sequences $\left(x_{n}\right)_{n=1}^{\infty}$ in $X$ such that $\left(\sum_{k=1}^{n}\left|x_{k}\right|^{2}\right)^{1 / 2}$ is order-bounded (and hence is a convergent sequence) in $X . X\left(\ell_{2}\right)$ becomes an order-continuous Banach lattice when normed by $\left\|\left(x_{n}\right)\right\|=\left\|\left(\sum_{n=1}^{\infty}\left|x_{n}\right|^{2}\right)^{1 / 2}\right\|$.

If $X$ has nontrivial cotype then $X\left(\ell_{2}\right)$ is naturally isomorphic to the space $\operatorname{Rad} X$ which is the subspace of $L_{2}([0,1] ; X)$ of functions of the form $\sum_{n=1}^{\infty} x_{n} r_{n}$ where $\left(r_{n}\right)$ is the sequence of Rademacher functions. The space $\operatorname{Rad} X$ is clearly an isomorphic invariant of $X$; so if two Banach lattices $X$ and $Y$ with nontrivial cotype are isomorphic, it follows easily that $X\left(\ell_{2}\right)$ and $Y\left(\ell_{2}\right)$ are isomorphic. However, this result holds in general by a result of Krivine [13] or [16, Theorem 1.f.14].

Theorem 2.1. If $X, Y$ are order-continuous Banach lattices and $T: X \longrightarrow Y$ is a bounded linear operator, then if $\left(x_{n}\right) \in X\left(\ell_{2}\right)$ we have $\left(T x_{n}\right) \in Y\left(\ell_{2}\right)$ and

$$
\left\|\left(T\left(x_{n}\right)\right)\right\|_{Y\left(\ell_{2}\right)} \leq K_{G}\|T\|\left\|\left(x_{n}\right)\right\|_{X\left(\ell_{2}\right)} .
$$

Here, as usual, $K_{G}$ denotes the Grothendieck constant.

Proof. Essentially this is Krivine's theorem, but we do need to show that if $\left(x_{n}\right) \in X\left(\ell_{2}\right)$ then $\left(T x_{n}\right) \in Y\left(\ell_{2}\right)$. To see this we show that $\left(\sum_{k=1}^{n}\left|T x_{k}\right|^{2}\right)^{1 / 2}$ is norm-Cauchy. In fact, if $m>n$ then

$$
\begin{gathered}
\left\|\left(\sum_{k=1}^{m}\left|T x_{k}\right|^{2}\right)^{1 / 2}-\left(\sum_{k=1}^{n}\left|T x_{k}\right|^{2}\right)^{1 / 2}\right\|_{Y} \leq\left\|\left(\sum_{k=n+1}^{m}\left|T x_{k}\right|^{2}\right)^{1 / 2}\right\|_{Y} \\
\leq K_{G}\|T\|\left\|\left(\sum_{k=n+1}^{m}\left|x_{k}\right|^{2}\right)^{1 / 2}\right\|_{X} \leq K_{G}\|T\|\left\|\left(\sum_{k=n+1}^{\infty}\left|x_{k}\right|^{2}\right)^{1 / 2}\right\|_{X}
\end{gathered}
$$

which converges to zero as $n \rightarrow \infty$ by the order-continuity of $X$.

Corollary 2.2. If two order-continuous Banach lattices $X$ and $Y$ are isomorphic as Banach spaces, then $X\left(\ell_{2}\right)$ and $Y\left(\ell_{2}\right)$ are isomorphic as Banach spaces.

If $X$ is a separable order-continuous nonatomic Banach lattice then $X$ can be represented as (i.e., is linearly and order isomorphic with) a Köthe function 
space on $[0,1]$ in such a way that $L_{\infty}[0,1] \subset X \subset L_{1}[0,1]$ and inclusions are continuous. It will then follow that $L_{\infty}$ is dense in $X$, and the dual of $X$ can be represented as a space of functions, namely, $X^{*}=\left\{f \in L_{1} \quad: \int|f g| d t<\right.$ $\infty$ for every $g \in X\}$.

Now we are ready to state our main result. Let us observe that for rearrangement invariant function spaces on $[0,1]$ this result was proved in [9] (cf. also $[16,2 . d]$ ) by a quite different technique.

Theorem 2.3. Let $X$ be an order-continuous, nonatomic Banach lattice with an unconditional basis. Then $X$ is isomorphic as a Banach space to $X\left(\ell_{2}\right)$.

Proof. We will represent $X$ as a Köthe function space on $[0,1]$ as described above. Suppose $\left(\phi_{n}\right)_{n=1}^{\infty}$ is a normalized unconditional basis of $X$. Then there is an order-continuous atomic Banach lattice $Y$ which we identify as a sequence space and operators $U: X \longrightarrow Y$ and $V: Y \longrightarrow X$ such that $U V=I_{Y}$, $V U=I_{X}$, and $U\left(\phi_{n}\right)=e_{n}$ for $n=1,2, \ldots$, where $e_{n}$ denotes the canonical basis vectors in $Y$. We can regard $Y^{*}$ as a space of sequences and further suppose that $\left\|e_{n}\right\|_{Y^{*}}=\left\|e_{n}\right\|_{Y}=1$. We will identify $Y\left(\ell_{2}\right)$ as a space of double sequences with canonical unconditional basis $\left(e_{m n}\right)_{m, n=1}^{\infty}$; thus for any finitely nonzero sequence we have $\left\|\sum a_{m n} e_{m n}\right\|_{Y\left(\ell_{2}\right)}=\left\|\sum_{m}\left(\sum_{n}\left|a_{m n}\right|^{2}\right)^{1 / 2} e_{m}\right\|_{Y}$.

Let $r_{n}$ denote the Rademacher functions and for each fixed $f \in X$ note that $\left(r_{n} f\right)$ converges weakly to zero, since for $g \in X^{*}$ we have $\lim _{n \rightarrow \infty} \int r_{n} f g d t=$ 0 . In particular, we have for each $m \in \mathbf{N}$ that $\left(r_{n} \phi_{m}\right)$ converges weakly to zero. It follows by a standard gliding hump technique that if $\eta=(2\|U\|\|V\|)^{-1}$ then we can find for each $(m, n) \in \mathbf{N}^{2}$ an integer $k(m, n)$ and disjoint subsets $\left(A_{m n}\right)$ of $\mathbf{N}$ so that $\left\|U\left(\phi_{m} r_{k(m, n)}\right) \chi_{A_{m n}}-U\left(\phi_{m} r_{k(m, n)}\right)\right\|_{Y} \leq \eta$.

Identifying $Y^{*}$ as a sequence space, we let $\psi_{m}=U^{*}\left(e_{m}\right)$ and then define $v_{m, n}=\chi_{A_{m n}} U\left(\phi_{m} r_{k(m, n)}\right) \in Y$ and $v_{m, n}^{*}=\chi_{A_{m n}} V^{*}\left(\psi_{m} r_{k(m, n)}\right) \in Y^{*}$. Now suppose $\left(a_{m n}\right)$ is a finitely nonzero double sequence. Then

$$
\begin{aligned}
\left\|\sum_{m, n} a_{m n} v_{m n}\right\|_{Y} & \leq\left\|\left(\sum_{m, n}\left|a_{m n}\right|^{2}\left|U\left(\phi_{m} r_{k(m, n)}\right)\right|^{2}\right)^{1 / 2}\right\|_{Y} \\
& \leq K_{G}\|U\|\left\|\left(\sum_{m, n}\left|a_{m n}\right|^{2}\left|\phi_{m} r_{k(m, n}\right|^{2}\right)^{1 / 2}\right\|_{X} \\
& =K_{G}\|U\|\left\|\left(\sum_{m}\left(\sum_{n}\left|a_{m n}\right|^{2}\right)\left|\phi_{m}\right|^{2}\right)^{1 / 2}\right\|_{X} \\
& =K_{G}\|U\|\left\|\left(\sum_{m}\left(\sum_{n}\left|a_{m n}\right|^{2}\right)\left|V e_{m}\right|^{2}\right)^{1 / 2}\right\|_{X} \\
& \leq K_{G}^{2}\|U\|\|V\|\left\|\left(\sum_{m}\left(\sum_{n}\left|a_{m n}\right|^{2}\right)\left|e_{m}\right|^{2}\right)^{1 / 2}\right\|_{Y} \\
& =K_{G}^{2}\|U\|\|V\|\left\|\sum_{m, n} a_{m n} e_{m n}\right\|
\end{aligned}
$$


Here we have used Krivine's theorem twice. It follows that we can define a linear operator $S: Y\left(\ell_{2}\right) \rightarrow Y$ by $S e_{m n}=v_{m n}$ and then $\|S\| \leq K_{G}^{2}\|U\|\|V\|$.

Similar calculations yield that for any finitely nonzero double sequence $\left(b_{m n}\right)$ we have

$$
\left\|\sum_{m, n} b_{m n} v_{m n}^{*}\right\|_{Y^{*}} \leq K_{G}^{2}\|U\|\|V\|\left\|\sum_{m}\left(\sum_{n}\left|b_{m n}\right|^{2}\right)^{1 / 2} e_{m}\right\|_{Y^{*}} .
$$

Suppose then $y \in Y$ and set $a_{m n}=\left\langle y, v_{m n}^{*}\right\rangle$. Let $F$ be a finite subset of $\mathbf{N}^{2}$. Let $\alpha_{m}=\left(\sum_{n} \chi_{F}(m, n)\left|a_{m n}\right|^{2}\right)^{1 / 2}$, and suppose the finitely nonzero sequence $\left(\beta_{m}\right)$ is chosen so that $\left\|\sum \beta_{m} e_{m}\right\|_{Y \cdot}=1$ and $\sum \beta_{m} \alpha_{m}=\left\|\sum \alpha_{m} e_{m}\right\|_{Y}$. Then, with the convention that $0 / 0=0$,

$$
\begin{aligned}
& \left\|\sum_{(m, n) \in F} a_{m n} e_{m n}\right\|_{Y\left(\ell_{2}\right)}=\sum_{m} \beta_{m} \alpha_{m} \\
& =\sum_{(m, n) \in F} \beta_{m} \alpha_{m}^{-1}\left|a_{m n}\right|^{2}=\left\langle y, \sum_{(m, n) \in F} \beta_{m} \alpha_{m}^{-1} a_{m n} v_{m n}^{*}\right\rangle \\
& \leq\|y\|_{Y}\left\|\sum_{(m, n) \in F} \beta_{m} \alpha_{m}^{-1} a_{m n} v_{m n}^{*}\right\|_{Y^{*}} \leq K_{G}^{2}\|U\|\|V\|\|y\|_{Y} .
\end{aligned}
$$

Thus for each $F$ the map $T_{F}: Y \rightarrow Y\left(\ell_{2}\right)$ given by

$$
T_{F} y=\sum_{(m, n) \in F}\left\langle y, v_{m n}^{*}\right\rangle e_{m n}
$$

has norm at most $K_{G}^{2}\|U\|\|V\|$. More generally, we have

$$
\left\|T_{F} y\right\| \leq K_{G}^{2}\|U\|\|V\|\left\|\chi_{A_{F}} y\right\|
$$

where $A_{F}=\bigcup_{(m, n) \in F} A_{m n}$.

It follows that for each $y \in Y$ the series $\sum_{m, n}\left\langle y, v_{m n}^{*}\right\rangle e_{m n}$ converges (unconditionally) in $Y\left(\ell_{2}\right)$. We can thus define an operator $T: Y \rightarrow Y\left(\ell_{2}\right)$ by $T y=\sum_{m, n}\left\langle y, v_{m n}^{*}\right\rangle e_{m n}$ and $\|T\| \leq K_{G}^{2}\|U\|\|V\|$.

Now notice that $T S\left(e_{m n}\right)=c_{m n} e_{m n}$ where $c_{m n}=\left\langle v_{m n}, v_{m n}^{*}\right\rangle$. But

$$
\begin{aligned}
\left\langle v_{m n}, v_{m n}^{*}\right\rangle & =\left\langle v_{m n}, V^{*} \psi_{m} r_{k(m, n)}\right\rangle \\
& \geq\left\langle U\left(\phi_{m} r_{k(m, n)}\right), V^{*}\left(\psi_{m} r_{k(m, n)}\right)\right\rangle-\eta\|V\|\left\|\psi_{m}\right\|_{X^{*}} \\
& =\left\langle\phi_{m}, \psi_{m}\right\rangle-\eta\|V\|\left\|\psi_{m}\right\|_{X^{*}} \geq 1-\eta\|V\|\|U\| \geq 1 / 2 .
\end{aligned}
$$

Thus TS is invertible, so it follows that $Y\left(\ell_{2}\right)$ is isomorphic to a complemented subspace of $Y$. It then follows from the Petczyński decomposition technique that $Y \sim Y\left(\ell_{2}\right)$; more precisely, $Y \sim Y\left(\ell_{2}\right) \oplus W$ for some $W$ and so $Y \sim$ $Y\left(\ell_{2}\right) \oplus\left(Y\left(\ell_{2}\right) \oplus W\right) \sim Y\left(\ell_{2}\right) \oplus Y \sim Y\left(\ell_{2}\right)$. The conclusion follows from Corollary 2.2 .

Remark. The order continuity of the Banach lattice $X$ is essential. In [14] a nonatomic Banach lattice $X$ (actually an M-space) was constructed which is isomorphic to $c_{0}$. In particular, $X$ has an unconditional basis but is not isomorphic to $X\left(\ell_{2}\right)$. 
Theorem 2.4. Let $Y$ be a Banach space with an unconditional basis. Then $Y$ is isomorphic to an order-continuous nonatomic Banach lattice if and only if $Y \sim Y\left(\ell_{2}\right)$.

Remark. Here again we regard $Y$ as an order-continuous Banach lattice.

Proof. One direction follows immediately from Theorem 2.3 and Corollary 2.2. For the other direction, it is only necessary to show that if $Y \sim Y\left(\ell_{2}\right)$ then $Y$ is isomorphic to order-continuous nonatomic Banach lattice. To this end we introduce the space $Y\left(L_{2}\right)$; this is the space of sequences of functions $\left(f_{n}\right)$ in $L_{2}[0,1]$ such that $\sum\left\|f_{n}\right\|_{2} e_{n}$ converges in $Y$. We set $\left\|\left(f_{n}\right)\right\|_{Y\left(L_{2}\right)}=$ $\left\|\sum\right\| f_{n}\left\|_{2} e_{n}\right\|_{Y}$. It is clear that $Y\left(L_{2}\right)$ is an order-continuous Banach lattice. Now if $\left(g_{n}\right)$ is an orthonormal basis of $L_{2}$, we define $W: Y\left(\ell_{2}\right) \rightarrow Y\left(L_{2}\right)$ by $W\left(\sum_{m, n} a_{m n} e_{m n}\right)=\left(\sum_{n} a_{m n} g_{n}\right)_{m=1}^{\infty}$, and it is easy to see that $W$ is an isometric isomorphism.

Proposition 2.5. If $X$ is a nonatomic order-continuous Banach lattice with unconditional basis, then $X \sim X \oplus X$ and $X \sim X \oplus R$.

Proof. Both facts follow from Theorem 2.3.

Note that for spaces with unconditional basis both properties do not hold in general (see $[5,6])$.

Proposition 2.6. Let $X$ be an order continuous nonatomic Banach lattice with an unconditional basis, and let $Y$ be a complemented subspace of $X$. Assume that $Y$ contains a complemented subspace isomorphic to $X$. Then $X \sim Y$.

Proof. The proof is a repetition of the proof of Proposition 2.d.5. of [16].

\section{HARDY SPACES}

We recall that $H_{1}\left(\mathbf{T}^{n}\right)$ is defined to be the space of boundary values of functions $f$ holomorphic in the unit disk $\mathbf{D}$ and such that

$$
\sup _{0<r<1} \int_{\mathbf{T}^{n}}\left|f\left(r e^{i t_{1}}, r e^{i t_{2}}, \ldots, r e^{i t_{n}}\right)\right| d t_{1} d t_{2} \ldots d t_{n}<\infty
$$

The basic theory of such spaces is explained in [18].

Let us consider first the case $n=1$. Then $\Re H_{1}$ is defined be the space of real functions $f \in L_{1}(\mathbf{T})$ such that for some $F \in H_{1}(\mathbf{T})$ we have $\Re F=f$. $\Re H_{1}$ is normed by $\|f\|_{1}+\min \left\{\|F\|_{H_{1}}: \Re F=f\right\}$. Then $H_{1}$ is isomorphic to the complexification of $\Re H_{1}$ and, further, when considered as a real space is isomorphic to $\Re H_{1}$. Further it was shown in [17] that $\Re H_{1}$ has an unconditional basis and is isomorphic to a space of martingales $H_{1}(\delta)$. To define the space $H_{1}(\delta)$ let $\left(h_{n}\right)_{n \geq 1}$ be the usual enumeration of the Haar functions on $I=[0,1]$ normalized so that $\left\|h_{n}\right\|_{\infty}=1$. Then suppose $f \in L_{1}$ is of the form $f=\sum a_{n} h_{n}$. We define $\|f\|_{H_{1}(\delta)}=\int\left(\sum_{n}\left|a_{n}\right|^{2} h_{n}^{2}\right)^{1 / 2} d t$ and $H_{1}(\delta)=\left\{f:\|f\|_{H_{1}(\delta)}<\infty\right\}$.

These considerations can be extended to the case $n>1$. In a similar way, $H_{1}\left(\mathbf{T}^{n}\right)$ is isomorphic to the complexification of, and is also real-isomorphic to, a martingale space $H_{1}\left(\delta^{n}\right)$. Here we define for $\alpha \in \mathscr{M}=\mathbf{N}^{n}$ the function $h_{\alpha} \in L_{1}\left(I^{n}\right)$ by $h_{\alpha}\left(t_{1}, \ldots, t_{n}\right)=\prod h_{\alpha_{k}}\left(t_{k}\right)$. Then $H_{1}\left(\delta^{n}\right)$ consists of all $f=$ $\sum_{\alpha \in \mathscr{M}} a_{\alpha} h_{\alpha}$ such that $\|f\|_{H_{1}\left(\delta^{n}\right)}=\int\left(\sum\left|a_{\alpha}\right|^{2} h_{\alpha}^{2}\right)^{1 / 2} d t<\infty$. 
It is clear from the definition that the system $\left(h_{\alpha}\right)_{\alpha \in \mathscr{M}}$ is an unconditional basis of $H_{1}\left(\delta^{n}\right)$. We can thus define a space $H_{1}\left(\delta^{n}, \ell_{2}\right)=H\left(\delta^{n}\right)\left(\ell_{2}\right)$ as in $\S 1$; since $H_{1}\left(\delta^{n}\right)$ has cotype two, this space is isomorphic to $\operatorname{Rad} H_{1}\left(\delta^{n}\right)$. The following theorem is due to Bourgain [2]:

Theorem 3.1. $H_{1}\left(\delta, \ell_{2}\right)$ is not isomorphic to a complemented subspace of $H_{1}(\delta)$.

In a subsequent paper [3] Bourgain implicitly extended this result to higher dimensions.

Theorem 3.2. For every $n=1,2, \ldots$ the space $H_{1}\left(\delta^{n}, \ell_{2}\right)$ is not isomorphic to any complemented subspace of $H_{1}\left(\delta^{n}\right)$.

Sketch of proof. For $n=1$ this theorem is proved in detail in [2]. The subsequent paper [3] states only the weaker fact that $H_{1}\left(\delta^{n}\right)$ is not isomorphic to $H_{1}\left(\delta^{n+1}\right)$. His proof, however, gives Theorem 3.2 as well. All that is needed is to change in $\S 3$ of [3] condition $(m+1)$ and Lemma 4. Before we formulate the appropriate condition we need some further notation. By $B M O\left(\delta^{n}\right)$ we will denote the dual of $H_{1}\left(\delta^{n}\right)$ and by $B M O\left(\delta^{n}, \ell_{2}\right)$ we will denote the dual of $H_{1}\left(\delta^{n}, \ell_{2}\right)$. The space $H_{1}\left(\delta^{n}, \ell_{2}\right)$ has an unconditional basis given by $\left(h_{\alpha} \otimes e_{k}\right)_{\alpha \in \mathscr{M}, k \in \mathrm{N}}$. In our notation from $\S 2 h_{\alpha} \otimes e_{k}$ is a sequence of $H_{1}\left(\delta^{n}\right)$ functions which consists of zero functions except at the $k$ th place where there is $h_{\alpha}$. The same element can be treated as an element of the dual space. Note that the natural duality gives

$$
\left\langle h_{\alpha} \otimes e_{k}, h_{\alpha^{\prime}} \otimes e_{k^{\prime}}\right\rangle= \begin{cases}\int_{I^{n}}\left|h_{\alpha}\right| & \text { when } \alpha=\alpha^{\prime} \text { and } k=k^{\prime}, \\ 0 & \text { otherwise. }\end{cases}
$$

Now we are ready to state the new condition $(m+1)$ :

Let $\Phi: H_{1}\left(\delta^{n}, \ell_{2}\right) \longrightarrow H_{1}\left(\delta^{n}\right)$ and $\Phi^{\times}: B M O\left(\delta^{n}, \ell_{2}\right) \longrightarrow B M O\left(\delta^{n}\right)$ be bounded linear operators (note that $\Phi^{\times}$is not the adjoint of $\Phi$ ). Then for every $\varepsilon>0$ there exists a set $A \subset \mathscr{M}$ such that $\sum_{\alpha \in A}\left|h_{\alpha}\right|=1$ and integers $k_{\alpha}$ for $\alpha \in A$ such that

$$
\sum_{\alpha \in A} \int_{I^{n}}\left|\Phi\left(h_{\alpha} \otimes e_{k_{\alpha}}\right)\right| \cdot\left|\Phi^{\times}\left(h_{\alpha} \otimes e_{k_{\alpha}}\right)\right|<\varepsilon .
$$

With this condition one can repeat the proof from [3] and obtain the theorem.

Corollary 3.3. We have

$$
\ell_{2} \stackrel{\complement}{\complement} H_{1}(\delta) \stackrel{\complement}{\complement} H_{1}\left(\delta, \ell_{2}\right) \stackrel{c}{\complement} H_{1}\left(\delta^{2}\right) \stackrel{c}{\complement} H_{1}\left(\delta^{2}, \ell_{2}\right) \stackrel{c}{\complement} \ldots
$$

where $X \stackrel{c}{\subset} Y$ means that $X$ is isomorphic to a complemented subspace of $Y$ but $Y$ is not isomorphic to a complemented subspace of $X$.

Proof. It is well known and easy to check that the map $h_{\alpha} \otimes e_{k} \mapsto h_{\alpha}\left(t_{1}, \ldots, t_{n}\right)$. $r_{k}\left(t_{n+1}\right)$ where $r_{k}$ is the $k$ th Rademacher function gives the desired complemented embedding. That no smaller space is isomorphic to a complemented subspace of a bigger one is the above theorem of Bourgain.

Corollary 3.4. The spaces $H_{1}\left(\delta^{n}\right)$ is not isomorphic to a nonatomic Banach lattice for $n=1,2, \ldots$. The spaces $H_{1}\left(\delta^{n}, \ell_{2}\right)$ are each isomorphic to a nonatomic Banach lattice. 
Proof. The first claim follows directly from Theorems 3.1, 3.2, and 2.3. We only have to observe that (since $H_{1}\left(\delta^{n}\right)$ does not contain any subspace isomorphic to $c_{0}$ and indeed has cotype two) any Banach lattice isomorphic as a Banach space to $H_{1}\left(\delta^{n}\right)$ is order continuous (see [16, Theorem 1.c.4]). The second claim follows from Corollary 2.4 .

Remark. For $H_{p}\left(\mathbf{T}^{n}\right)$ with $0<p<\infty$ we have the following situation. When $1<p<\infty$ the orthogonal projection from $L_{p}\left(\mathbf{T}^{n}\right)$ onto $H_{p}\left(\mathbf{T}^{n}\right)$ is bounded so then $H_{p}\left(\mathbf{T}^{n}\right)$ is isomorphic to $L_{p}\left(\mathbf{T}^{n}\right)$. This implies in particular that these spaces are isomorphic to nonatomic lattices. When $0<p<1$ then $H_{p}\left(\mathbf{T}^{n}\right)$ admit only purely atomic orders as a $p$-Banach lattices. To see this observe that if $X$ is not a purely atomic $p$-Banach lattice then its Banach envelope (for definition and properties see [11]) is a Banach lattice which is not purely atomic. On the other hand it is known that the Banach envelope of $H_{p}\left(\mathbf{T}^{n}\right)$ is isomorphic to $\ell_{1}$. For $n=1$ this can be found in [11, Theorem 3.9], for $n>1$ the proof uses [19, Theorem $\left.2^{\prime}\right]$ but otherwise is the same; alternatively see [11, Theorem 3.5] for a proof using bases. When we compare it with the observation from [1] mentioned in the Introduction, that $\ell_{1}$ is not isomorphic to any nonatomic Banach lattice, we conclude that the spaces $H_{p}\left(\mathbf{T}^{n}\right)$ cannot be isomorphic to any nonatomic $p$-Banach lattice.

Remark. For the dual spaces $H_{1}\left(\mathbf{T}^{n}\right)^{*}=B M O\left(\mathbf{T}^{n}\right)$ the situation is rather different. We first observe the following proposition:

Proposition 3.5. For any Banach space $X$ the spaces $\ell_{1}(X)^{*}\left(=\ell_{\infty}\left(X^{*}\right)\right)$ and $L_{1}([0,1], X)^{*}$ are isomorphic.

Proof. Clearly $\ell_{1}(X)^{*}$ is isomorphic to a one-complemented subspace of $L_{1}(X)^{*}$. Now let $\chi_{n, k}=\chi_{\left((k-1) 2^{-n}, k 2^{-n}\right)}$ for $1 \leq k \leq 2^{n}$ and $n=0,1, \ldots$ Let $T: \ell_{1}(X) \rightarrow L_{1}(X)$ be defined by $T\left(\left(x_{n}\right)\right)=\sum x_{n} \chi_{m, k}$ where $n=2^{m}+k-1$. Let $L_{1}\left(\mathscr{D}_{N}, X\right)$ be the subspace of all functions measurable with respect to the finite algebra generated by the sets $\left((k-1) 2^{-N}, k 2^{-N}\right)$ for $1 \leq k \leq 2^{N}$, and define $S_{N}: L_{1}\left(\mathscr{D}_{N} ; X\right) \rightarrow \ell_{1}(X)$ by setting $S\left(x \otimes \chi_{N, k}\right)$ to be the element with $x$ in position $2^{N}+k-1$ and zero elsewhere. Then applying [22, II.E, Exercise 7] (cf. [8, Proposition 1]), we obtain that $L_{1}(X)^{*}$ is isomorphic to a complemented subspace of $\ell_{1}(X)^{*}$. Then by the Petczyński decomposition technique we obtain the proposition.

Now from Proposition 3.5, observe that, since $H_{1}\left(\mathbf{T}^{n}\right) \sim \ell_{1}\left(H_{1}\left(\mathbf{T}^{n}\right)\right)$, we have $L_{1}\left(H_{1}\left(\mathbf{T}^{n}\right)\right)^{*} \sim B M O\left(\mathbf{T}^{n}\right)$, and clearly this isomorphism induces a nonatomic (but not order-continuous) lattice structure on $B M O\left(T^{n}\right)$. (It is easy to see that a space which contains a copy of $\ell_{\infty}$ cannot have an order-continuous lattice structure, because it fails the separable complementation property.)

\section{4. $\operatorname{Rad} H_{1}$ AND TENT SPACES}

The space $H_{1}\left(\delta, \ell_{2}\right)$ is, as observed in $\S 2$, isomorphic to $\operatorname{Rad} H_{1}$ and has a structure as a nonatomic Banach lattice. The complex space $\operatorname{Rad} H_{1}$ is easily seen to be isomorphic to the vector-valued space $H_{1}\left(\mathbf{T}, \ell_{2}\right)$ consisting of the boundary values of the space of all functions $F$ analytic in the unit disk D 
with values in a Hilbert space $\ell_{2}$ and such that

$$
\sup _{0<r<1} \int_{0}^{2 \pi}\left\|F\left(r e^{i \theta}\right)\right\| \frac{d \theta}{2 \pi}=\|F\|<\infty .
$$

To see this isomorphism just note that $H_{1}\left(\mathbf{T}, \ell_{2}\right)$ can be identified with the space of sequences $\left(f_{n}\right)$ in $H_{1}$ such that

$$
\left\|\left(f_{n}\right)\right\|=\int_{0}^{2 \pi}\left(\sum_{n=1}^{\infty}\left|f_{n}\left(e^{i \theta}\right)\right|^{2}\right)^{1 / 2} \frac{d \theta}{2 \pi}<\infty .
$$

This is in turn easily seen to be equivalent to the norm of $\sum r_{n} f_{n}$ in $L_{2}\left([0,1] ; H_{1}\right)$ (see $[16$, Theorem 1.d.6]).

We now show that a nonatomic Banach lattice isomorphic to $\operatorname{Rad} H_{1}$ arises naturally in in harmonic analysis. More precisely we will show that tent space $T^{1}$, which was introduced and studied by Coifman, Meyer, and Stein in [4], is isomorphic to $\operatorname{Rad} H_{1}$. Tent spaces are useful in some questions of harmonic analysis (cf. [7] or [21]). They can be defined over $\mathbf{R}^{n}$, but for the sake of simplicity we will consider them only over $\mathbf{R}$.

Let us fix $\alpha>0$. For $x \in \mathbf{R}$ we define

$$
\Gamma_{\alpha}(x)=\left\{(y, t) \in \mathbf{R} \times \mathbf{R}^{+}:|x-y|<\alpha t\right\} .
$$

Given a function $f(y, t)$ defined on $\mathbf{R} \times \mathbf{R}^{+}$we put

$$
\|f\|_{\alpha}=\int_{\mathbf{R}}\left(\int_{\Gamma_{\alpha}(x)}|f(y, t)|^{2} t^{-2} d y d t\right)^{1 / 2} d x .
$$

It was shown in [4, Proposition 4] that for different $\alpha$ 's the norms $\|\cdot\|_{\alpha}$ are equivalent; i.e., for $0<\alpha<\beta<\infty$ there is a $C=C(\alpha, \beta)$ such that for every $f$ we have

$$
\|f\|_{\alpha} \leq\|f\|_{\beta} \leq C\|f\|_{\alpha} .
$$

This implies that the space $T^{1}=\left\{f(y, t):\|f\|_{\alpha}<\infty\right\}$ does not depend on $\alpha$. Observe that $T^{1}$ is clearly a nonatomic Banach lattice.

The main result of this section is

Theorem 4.1. The space $T^{1}$ is lattice-isomorphic to $H_{1}\left(\delta, L_{2}\right)$ and, hence, isomorphic to $\operatorname{Rad} H_{1}$.

Actually for the proof of this theorem it is natural to work with the dyadic $H_{1}$ space on $\mathbf{R}$. This space, which we denote $H_{1}\left(\delta_{\infty}\right)$, can be defined as follows:

Let $I_{n k}=\left[k \cdot 2^{n},(k+1) \cdot 2^{n}\right]$ for $n, k=0, \pm 1, \pm 2 \ldots$, and let $h_{n k}$ be the function which is equal to 1 on the left-hand half of $I_{n k},-1$ on the right-hand half of $I_{n k}$, and zero outside $I_{n k}$. In other words, $h_{n k}$ is the Haar system on R. The system $\left\{h_{n k}\right\}_{n, k=0, \pm 1, \pm 2, \ldots}$ is a complete orthogonal system. For a function $f=\sum_{n, k} a_{n k} h_{n k}$ we define its $H_{1}\left(\delta_{\infty}\right)$-norm by

$$
\|f\|=\int_{\mathbf{R}}\left(\sum_{n, k}\left|a_{n k}\right|^{2}\left|h_{n k}\right|^{2}\right)^{1 / 2} d t .
$$

That this space is isomorphic to the space $H_{1}(\delta)$ follows from the work of Sjölin and Stromberg [20]. However, slightly more is true: 
Lemma 4.2. The atomic Banach lattices $H_{1}(\delta)$ and $H_{1}\left(\delta_{\infty}\right)$ are lattice-isomorphic (or, equivalently the natural normalized unconditional bases of these spaces are permutatively equivalent).

Proof. For any subset $\mathscr{A}$ of $\mathbf{Z}^{2}$ write $H_{\mathscr{A}}$ for the closed linear span of $\left\{h_{n k}\right.$ : $(n, k) \in \mathscr{A}\}$ in $H_{1}\left(\delta_{\infty}\right)$. For $m \in \mathbf{Z}$ let $\mathscr{A}_{m}=\left\{(n, k): I_{n k} \subset\left[2^{-m-1}, 2^{-m}\right]\right\}$ and $\mathscr{B}_{m}=\left\{(n, k): I_{n k} \subset\left[-2^{-m},-2^{-m-1}\right]\right.$. Let $\mathscr{D}=\bigcup_{m \in Z}\left(\mathscr{A}_{m} \cup \mathscr{B}_{m}\right)$ and $\mathscr{D}_{+}=\bigcup_{m>0} \mathscr{A}_{m}$. Then it is clear that $H_{\mathscr{D}}$ and $H_{\mathscr{D}_{+}}$are each lattice isomorphic to $\ell_{1}\left(H_{1}(\delta)\right)$. Now $H_{1}\left(\delta_{\infty}\right)$ is lattice isomorphic to $H_{\mathscr{D}} \oplus H_{\mathscr{E}}$ where $\mathscr{E}=$ $\{(m, 0),(m,-1): m \in \mathbf{Z}\}$. It is easy to show that $H_{\mathscr{E}}$ is lattice isomorphic to $\ell_{1}$. Similarly $H_{1}(\delta)$ is lattice-isomorphic to $H_{1}\left(\mathscr{D}_{+}\right) \oplus \ell_{1}$, and this completes the proof of the lemma.

Remark. Note also that $H_{1}(\delta)$ is lattice-isomorphic to $\ell_{1}\left(H_{1}(\delta)\right)$.

Proof of Theorem 4.1. We will prove that $T^{1}$ is lattice-isomorphic to $H_{1}\left(\delta_{\infty}, L_{2}\right)$. Let us introduce squares $A_{n k} \subset \mathbf{R} \times \mathbf{R}^{+}$defined as $A_{n k}=$ $I_{n k} \times\left[2^{n}, 2^{n+1}\right]$ for $n, k=0, \pm 1, \pm 2, \ldots$. It is geometrically clear that squares $\left\{A_{n k}\right\}_{n, k=0, \pm 1, \pm 2, \ldots}$ are essentially disjoint and that they cover $\mathbf{R} \times \mathbf{R}^{+}$. For $j=0,1,2$ we define

$$
A_{n k}^{j}=\left[(k+j / 3) 2^{n},(k+(j+1) / 3) 2^{n}\right] \times\left[2^{n}, 2^{n+1}\right] .
$$

Note that in this way we divide each $A_{n k}$ into three essentially disjoint rectangles. Let $D^{j}=\bigcup_{n, k} A_{n k}^{j}$. Let $T_{j}^{1}$ be the subspace of $T^{1}$ consisting of all functions whose support is contained in $D^{j}$. Clearly $T^{1}=T_{0}^{1} \oplus T_{1}^{1} \oplus T_{2}^{1}$, so it is enough to show that $T_{j}^{1}$ is lattice-isomorphic to $H_{1}\left(\delta_{\infty}, L_{2}\right)$.

We write $f^{j} \in T_{j}^{1}$ as $f^{j}=\sum_{n, k} f_{n k}^{j}$ where $f_{n k}^{j}=f^{j} \cdot \chi_{A_{n k}^{j}}$. We start with $j=1$. For any $\alpha>0$ we have

$$
\begin{aligned}
\left\|f^{1}\right\|_{\alpha} & =\int_{\mathbf{R}}\left(\int_{\Gamma_{\alpha}(x)}\left|f^{1}(y, t)\right|^{2} t^{-2} d y d t\right)^{1 / 2} d x \\
& =\int_{\mathbf{R}}\left(\int_{\Gamma_{\alpha}(x)} \sum_{n, k}\left|f_{n k}^{1}(y, t)\right|^{2} t^{-2} d y d t\right)^{1 / 2} d x \\
& =\int_{\mathbf{R}}\left(\sum_{n k} \int_{\Gamma_{\alpha}(x)}\left|f_{n k}^{1}(y, t)\right|^{2} t^{-2} d y d t\right)^{1 / 2} d x .
\end{aligned}
$$

If we now take $\alpha=\frac{2}{3}$ we have $\Gamma_{\alpha}(x) \supset A_{n k}^{1}$ for all $x \in I_{n k}$, so from (4.3) we get

$$
\left\|f^{1}\right\|_{\alpha} \geq \int_{\mathbf{R}}\left(\sum_{n k} \chi_{I_{n k}}(x) \int_{A_{n k}^{1}}\left|f_{n k}^{1}(y, t)\right|^{2} t^{-2} d y d t\right)^{1 / 2} d x .
$$

On the other hand, when we take $\alpha=\frac{1}{6}$ we have $\Gamma_{\alpha}(x) \cap A_{n k}^{1}=\varnothing$ for all $x \notin I_{n k}$, so from (4.3) we get

$$
\left\|f^{1}\right\|_{\alpha} \leq \int_{\mathbf{R}}\left(\sum_{n, k} \chi_{I_{n k}}(x) \int_{A_{n k}^{1}}\left|f_{n k}^{1}(y, t)\right|^{2} t^{-2} d y d t\right)^{1 / 2} d x .
$$


For each $(n, k)$ the subspace of $T^{1}$ consisting of functions supported on $A_{n k}^{1}$ is easily seen to be isometric to the Hilbert space. If we fix an isometry between this space and $\ell_{2}$, we obtain from (4.2)-(4.4) that $T_{1}^{1}$ is lattice-isomorphic to $H_{1}\left(\delta_{\infty}, L_{2}\right)$. In order to complete the proof of Theorem 4.1 it is enough to show that $T_{0}^{1}$ and $T_{2}^{1}$ are lattice-isomorphic to $T_{1}^{1}$. This isomorphism can be given by $\sum_{n k} f_{n k}^{j} \mapsto \sum_{n k} f_{n k}^{1}$. The fact that this map is really an isomorphism follows from

Lemma 4.3. Let $\phi(t)$ be a uniformly bounded measurable function on $\mathbf{R}^{+}$. For a function $f$ defined on $\mathbf{R} \times \mathbf{R}^{+}$we define

$$
A_{\phi}(f)(y, t)=f(y+t \phi(t), t) .
$$

Then $A_{\phi}: T^{1} \longrightarrow T^{1}$ is a continuous linear operator.

Proof of Lemma 4.3. Since

$$
\begin{aligned}
\int_{\Gamma_{\alpha}(x)} & \left|A_{\phi}(f)(y, t)\right|^{2} t^{-2} d y d t=\int_{\mathbf{R}^{+}}\left(t^{-2} \int_{x-\alpha t}^{x+\alpha t}\left|A_{\phi}(f)(y, t)\right|^{2} d y\right) d t \\
= & \int_{\mathbf{R}^{+}}\left(t^{-2} \int_{x-\alpha t-t \phi(t)}^{x+\alpha t-t \phi(t)}|f(y, t)|^{2} d y\right) d t \\
\leq & \int_{\mathbf{R}^{+}}\left(t^{-2} \int_{x-\left(\|\phi\|_{\infty}+\alpha\right) t}^{x+\left(\|\phi\|_{\infty}+\alpha\right) t}|f(y, t)|^{2} d y\right) d t \\
= & \int_{\Gamma_{\alpha+\|\phi\| \|_{\infty}}(x)}|f(y, t)|^{2} t^{-2} d y d t,
\end{aligned}
$$

the lemma follows.

\section{REFERENCES}

1. Ju. A. Abramovič and P. Wojtaszczyk, On the uniqueness of order in the spaces $\ell_{p}$ and $L_{p}[0,1]$, Mat. Zametki 18 (1975), 313-325.

2. J. Bourgain, Non-isomorphism of $H^{1}$-spaces in one and several variables, J. Funct. Anal. 46 (1982), 45-57.

3. Belg. Ser. B 35 (1983), 127-136.

4. R. R. Coifman, Y. Meyer, and E. Stein, Some new function spaces and their applications to harmonic analysis, J. Funct. Anal. 62 (1985), 304-335.

5. T. Figiel, An example of an infinite dimensional, reflexive Banach space non-isomorphic to its Cartesian square, Studia Math. 42 (1972), 295-306.

6. W. T. Gowers, A solution to Banach's hyperplane problem (to appear).

7. E. Harboure, J. L. Torrea and B. E. Viviani, $A$ vector valued approach to tent spaces, J. Analyse Math. 66 (1991), 125-140.

8. W. B. Johnson, A complementably universal conjugate Banach space and its relation to the approximation property, Israel J. Math. 13 (1972), 301-310.

9. W. B. Johnson, B. Maurey, G. Schechtman, and L. Tzafriri, Symmetric structures in Banach spaces, Mem. Amer. Math. Soc., No. 217, Amer. Math. Soc., Providence, RI, 1979.

10. N. J. Kalton, Lattice structures on Banach spaces, Mem. Amer. Math. Soc., no. 493, vol. 103, Amer. Math. Soc., Providence, RI, 1993.

11. N. J. Kalton, C. Leranoz, and P. Wojtaszczyk, Uniqueness of unconditional bases in quasiBanach spaces with applications to Hardy spaces, Israel J. Math. 72 (1990), 299-311. 
12. N. J. Kalton, N. T. Peck, and J. W. Roberts, An F-space sampler, London Math. Soc. Lecture Note Ser., vol. 89, Cambridge Univ. Press, Cambridge, 1984.

13. J. L. Krivine, Théorèmes de factorisation dans les espaces réticules, Seminaire Maurey-Schwartz 1973-74, Exposes 22-23, École Polytechnique, Paris.

14. E. Lacey and P. Wojtaszczyk, Nonatomic Banach lattices can have $\ell_{1}$ as a dual space, Proc. Amer. Math. Soc. 57 (1976), 79-84.

15. J. Lindenstrauss and L. Tzafriri, Classical Banach spaces. I. Sequence spaces, SpringerVerlag, Berlin, 1977.

16. __ Classical Banach spaces. II. Function spaces, Springer-Verlag, Berlin, 1979.

17. B. Maurey, Isomorphismes entre espaces $H_{1}$, Acta Math. 145 (1980), 79-120.

18. W. Rudin, Function theory in polydiscs, Benjamin, New York, 1969.

19. J. H. Shapiro, Mackey topologies, reproducing kernels, and diagonal maps on the Hardy and Bergman spaces, Duke Math. J. 43.1 (1976), 187-202.

20. P. Sjölin and J.-O. Stromberg, Basis properties of Hardy spaces, Ark. Mat. 21 (1983), 111-125.

21. A. Torchinsky, Real-variable methods in harmonic analysis, Academic Press, New York, 1986.

22. P. Wojtaszczyk, Banach spaces for analysts, Cambridge Stud. Adv. Math., vol. 25, Cambridge Univ. Press, Cambridge, 1991.

Department of Mathematics, University of Missouri, Columbia, Missouri 65211

E-mail address: MATHNJK QMIZZOU1. BITNET

Institut of Mathematics, Warsaw University, 00-913 Warszawa, ul. Banacha 2, POLAND

E-mail address: PRZEMEKOMIMUW. EDU.PL 